\title{
The problem of dynamic cavitation in nonlinear elasticity
}

\author{
(In: Séminaire Laurent Schwartz - EDP et applications (2012-2013), Exp. 14, 1-17. DOI: 10.5802/slsedp.41.)
}

\author{
Jan Giesselmann* $\quad$ Alexey Miroshnikov ${ }^{\dagger} \quad$ Athanasios E. Tzavaras ${ }^{\ddagger}$
}

\begin{abstract}
The notion of singular limiting induced from continuum solutions (slic-solutions) is applied to the problem of cavitation in nonlinear elasticity, in order to re-assess an example of non-uniqueness of entropic weak solutions (with polyconvex energy) due to a forming cavity.
\end{abstract}

\section{Introduction}

The equations describing radial motions of isotropic elastic materials admit a special weak solution describing a cavity that emerges at a point from a homogeneously deformed state. Cavitating solutions are self-similar in nature and were introduced by Ball [3] and by Pericak-Spector and Spector [11, 12]. They turn out to decrease the total mechanical energy and provide a striking example of non-uniqueness of entropy weak solutions for polyconvex energies [11.

There is a class of problems in solid mechanics, such as fracture, cavitation or shear bands, where discontinuous motions emerge from smooth motions via a mechanism of material instability. Any attempt to study such solutions that lie at the limits of continuum modeling needs to reckon with the problem of giving a proper definition for such solutions. Once the material breaks or a shear band forms the motion can no longer be described at the level of continuum modeling and microscopic modeling or higher-order regularizing mechanisms have to be taken into account. Still, as such structures develop there is expected an intermediate time scale where both types of modeling apply. In these lecture notes we present the notion of singular limiting induced from continuum solution (or slic-solution) [8, stating that a discontinuous motion is a slic-solution if its averages are a family of smooth approximate solutions to the problem.

The lecture notes are based on two recent works on cavity formation [10] and [8]. [10] complements [11, 12] and establishes various further properties of weak solutions describing cavitation, in particular indicating that cavity formation is necessarily associated with a unique precursor shock. In [8] the notion of singular limiting induced from continuum solution is introduced and applied to the problem of cavity formation. It is shown that there is an energetic cost for creating the cavity, which is captured by slic-solutions but neglected by the usual entropic weak solutions. Once this surface energy cost is accounted for, the paradox of nonuniqueness is removed, in the sense that

\footnotetext{
*Weierstrass Institute, Berlin, Germany, jan.giesselmann@wias-berlin.de

${ }^{\dagger}$ Department of Mathematics and Statistics, University of Massachusetts Amherst, USA, amiroshn@gmail.com

${ }^{\ddagger}$ Department of Applied Mathematics, University of Crete, Heraklion, Greece and Institute for Applied and Computational Mathematics, FORTH, Heraklion, Greece, tzavaras@tem.uoc.gr
} 
the cavitating solution together with the surface energy of the cavity has higher energy than the homogeneous deformation.

The structure of these notes is as follows: In section 2 we present the system of elasticity and the general requirements imposed by mechanical considerations and discuss various notions of relaxed convexity hypotheses associated to elasticity. In section 3 we introduce the equations of radial elasticity applicable to isotropic elastic materials. In section 4 we present the problem of cavitation and outline the construction and properties of weak solutions describing cavity formation from a homogeneously deformed state. In section 5 we introduce an illuminating example for fracture in 1-d and in section 6 the concept of singular limiting induced from continuum solutions which gives an interpretation to this example as a solution of the equations of one-dimensional elasticity. It is shown that there is an additional contribution from the opening crack to the resulting energy of the fracturing solution. In section 7 , the notion of slic-solution is extended for the cavitation problem and the effect of this concept to the energy of the cavitating solution is calculated. It turms out that the slic-solution provides a more discriminating concept of solution for strong singularities.

\section{The equations of elasticity}

The equations of elasticity is the system of nonlinear partial differential equations

$$
\frac{\partial^{2} y}{\partial t^{2}}=\operatorname{div} \frac{\partial W}{\partial F}(\nabla y)
$$

where $y(x, t): \mathbb{R}^{d} \times \mathbb{R}_{+} \rightarrow \mathbb{R}^{d}$ describes the motion. It is customary to introduce the velocity $v=\frac{\partial y}{\partial t}$ and the deformation gradient $F=\nabla y$ and to write (2.1) as a first order hyperbolic system

$$
\left\{\begin{array}{c}
\partial_{t} F_{i \alpha}=\partial_{\alpha} v_{i} \\
\partial_{t} v_{i}=\partial_{\alpha} \frac{\partial W}{\partial F_{i \alpha}}(F) \\
\partial_{\alpha} F_{i \beta}-\partial_{\beta} F_{i \alpha}=0
\end{array}\right.
$$

The last equation stands for a constraint that ensures that $F$ is a gradient. This constraint does not cause problems as it is an involution [4, that is if it is satisfied for the initial data then it is propagated by $(2.2)_{1}$ to the solutions. In (2.1) the hypothesis of hyperelasticity, $S=\frac{\partial W}{\partial F}$, is used stating that the Piola-Kirchhoff stress $S$ is derived as a gradient of a stored energy function $W$ : $M^{d \times d} \rightarrow \mathbb{R}$. This hypothesis makes the theory consistent with the second law of thermodynamics.

The principle of frame indifference dictates that the stored energy satisfies the invariance

$$
W(Q F)=W(F) \quad \forall Q \in S O(d)
$$

for any matrix $Q$ describing a proper rotation. In order to interpret a geometric map $y$ as a physical motion, $y$ has to be globally one-to-one so that matter does not interpenetrate. A necessary condition for achieving that is to cost infinite energy whenever a finite volume is compressed down to a zero volume, what dictates the natural assumption for the stored energy

$$
W(F) \rightarrow \infty \quad \text { as } \quad \operatorname{det} F \rightarrow 0 .
$$

These two requirements are in general inconsistent with convexity of the stored energy as can be seen in the schematic picture of Figure 1. It is thus too restrictive to take $W$ as a convex function. 


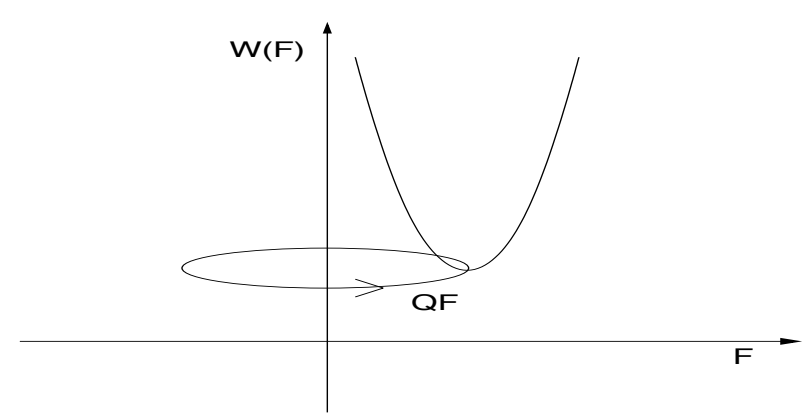

Figure 1: A depiction of the stored energy

The system $(2.2)$ is a system of conservation laws. It is equipped for smooth solutions with the identity

$$
\partial_{t}\left(\frac{1}{2}|v|^{2}+W(F)\right)-\partial_{\alpha}\left(v_{i} \frac{\partial W}{\partial F_{i \alpha}}(F)\right)=0
$$

expressing the conservation of mechanical energy. In the parlance of conservation law $\eta=\frac{1}{2}|v|^{2}+$ $W(F)$ is an entropy with associated entropy flux $q_{\alpha}=-v_{i} \frac{\partial W}{\partial F_{i \alpha}}$. The issue in the background is that, due to the lack of convexity of $W$, the entropy $\eta$ is not convex. On the other hand the standard theory of systems of conservation laws is intricately connected to convexity and is inapplicable in a direct way. Hyperbolicity of 2.2 is equivalent to the condition on $W$ :

$$
\frac{\partial^{2} W}{\partial F_{i \alpha} \partial F_{j \beta}}(F) \xi_{i} \xi_{j} \nu_{\alpha} \nu_{\beta}>0 \quad \forall \xi \neq 0, \nu \in \mathcal{S}^{d-1}
$$

called Legendre-Hadamard conditions and associated to positive-definiteness of the acoustic tensor.

A lot of effort has been devoted into finding suitable notions to replace the assumption of convexity, starting from works on the calculus of variations associated to the minimization of potential energy. The following notions have emerged: $W(F)$ is called (strictly) rank-1 convex if

$$
W(\theta F+(1-\theta) G)<\theta W(F)+(1-\theta) W(G) \quad 0<\theta<1, \forall F, G \quad \text { with } F-G=\xi \otimes \nu .
$$

Strict rank-1 convexity is equivalent to the Legendre-Hadamard conditions for $W$ sufficiently smooth. $W(F)$ is called polyconvex if it is expressed in the form

$$
W(F)=g(F, \operatorname{cof} F, \operatorname{det} F)=g \circ \Phi(F) \quad \text { with } g(\Xi) \text { convex. }
$$

The latter definition is intimately connected to the notion of null-Lagrangean which is the analog of constant functions in the calculus of variations. An integrand $\Phi(F)$ is a null-Lagrangean iff

$$
\int_{\Omega} \Phi(\nabla y+\nabla \phi) d x=\int_{\Omega} \Phi(\nabla y) d x \quad \forall y \in W^{1, p}, \phi \in C_{c}^{\infty} .
$$

The following equivalences hold: 
$\Phi(F)$ is a null-Lagrangean

$$
\begin{aligned}
& \Longleftrightarrow \int_{\Omega} \Phi(F+\nabla \phi) d x=\Phi(F)|\Omega| \quad \forall F \in M^{d \times d}, \phi \in C_{c}^{\infty} \\
& \Longleftrightarrow \quad \Phi(F) \text { is rank-1 affine } \\
& \Longleftrightarrow \quad \Phi(F)=\alpha F+\beta \operatorname{cof} F+c \operatorname{det} F \\
& \Longleftrightarrow \partial_{\alpha}\left(\frac{\partial \Phi}{\partial F_{i \alpha}}(\nabla y)\right)=0 \quad \text { in } \mathcal{D}^{\prime}
\end{aligned}
$$

The reader is referred to [1, 2] for precise statements and their proofs, The introduction of nullLagrangeans into the theory of elasticity is due to Ericksen [7] and Edelen [6] and their role in the mathematical theory of elasticity is pointed out by Ball [1]. Their importance arises from the fact that null-Lagrangeans are weakly continuous in $W^{1, p}[2$, and from their role in the existence theory of equilibrium elasticity [1, 2] and in obtaining symmetrizable extensions for polyconvex elastodynamics [13, 15, 16].

Here, we explore their role in the problem of cavity formation.

\section{Radial motions in 3-d elasticity}

A motion of the form $y(x, t)=w(R, t) \frac{x}{R}$, where $R=|x|, x \in \mathbb{R}^{d}$, is called radial. For an elastic material to support radial motions it must be isotropic and as always frame indifferent, that is the stored energy satisfies

$$
W(Q F)=W(F)=W(F Q) \quad \forall Q \in S O(d)
$$

The class of such stored energies has been characterized to be of the form $W(F)=\Phi\left(v_{1}, v_{2}, \ldots, v_{d}\right)$, where $\Phi: \mathbb{R}_{++}^{d} \rightarrow \mathbb{R}$ is a symmetric function of the eigenvalues $v_{i}$ of $+\sqrt{F^{T} F}$ the so called principal stretches (see [15]).

For reasons that will be explained later we will restrict attention here to the case of three space dimensions $d=3$ and the special example of stored energy

$$
\Phi\left(v_{1}, v_{2}, v_{3}\right)=\frac{1}{2}\left(v_{1}^{2}+v_{2}^{2}+v_{3}^{2}\right)+h\left(v_{1} v_{2} v_{3}\right),
$$

with $h: \mathbb{R}_{+} \rightarrow \mathbb{R}_{+}$being a $C^{3}$ convex function that satisfies $h(\delta) \rightarrow+\infty$ as $\delta \rightarrow 0+$. This stored energy is of the polyconvex class, and the behavior as $\delta \rightarrow 0+$ is placed to avoid that a finite volume is compressed down to zero and guarantee that the solutions avoid infinite compression and can thus be interpreted as elastic motions.

\subsection{The equations of radial elasticity.}

For radial motions $y(x, t)=w(R, t) \frac{x}{R}$, with $R=|x|, x \in \mathbb{R}^{d}$, the deformation gradient is of the form

$$
F=w_{R} \hat{x} \otimes \hat{x}+\sum_{j=2}^{d} \frac{w}{R} \hat{x}_{j}^{\perp} \otimes \hat{x}_{j}^{\perp},
$$


where $\hat{x}=\frac{x}{R}, \hat{x}_{j}^{\perp}$ the perpendicular unit vectors, and the principal stretches are $w_{R}$ in the radial direction and $\frac{w}{R}$ of multiplicity $d-1$ in the orthogonal directions. The Piola-Kirchhoff stress tensor is computed as

$$
S=\frac{\partial W}{\partial F}=\frac{\partial \Phi}{\partial v_{1}} \hat{x} \otimes \hat{x}+\sum_{j=2}^{d} \frac{\partial \Phi}{\partial v_{j}} \hat{x}_{j}^{\perp} \otimes \hat{x}_{j}^{\perp} .
$$

To represent a physically realizable motion, we impose that $\operatorname{det} F=w_{R}(w / R)^{d-1}>0$ where $F=\nabla y$. This is equivalent to $w_{R}>0$ a condition that also suffices to avoid interpenetration of matter for radial motions.

A cumbersome but straightforward computation shows that $w(R, t)$ satisfies the partial differential equation of second-order

$$
w_{t t}=\frac{1}{R^{d-1}} \partial_{R}\left(R^{d-1} \frac{\partial \Phi}{\partial v_{1}}\left(w_{R}, \frac{w}{R}, \ldots, \frac{w}{R}\right)\right)-\frac{d-1}{R} \frac{\partial \Phi}{\partial v_{2}}\left(w_{R}, \frac{w}{R}, \ldots, \frac{w}{R}\right) .
$$

It is instructive to give a short alternative derivation of (3.1). Consider the action functional $\mathcal{L}=K-P$, where $K$ is the kinetic and $P$ the potential energy:

$$
\mathcal{L}[w]=K-P=\int_{0}^{T} \int_{0}^{1} \frac{1}{2} w_{t}^{2} R^{d-1}-\Phi\left(w_{R}, \frac{w}{R}, \ldots, \frac{w}{R}\right) R^{d-1} d R d t .
$$

The critical points of the functional $\mathcal{L}$ are computed by setting to zero the variational derivative $\left.\frac{d}{d \delta}\right|_{\delta=0} \mathcal{L}(w+\delta \psi)=0$. This leads to the Euler-Lagrange equations

$$
\int_{0}^{T} \int_{0}^{1} R^{d-1} w_{t} \psi_{t}-R^{d-1} \frac{\partial \Phi}{\partial v_{1}} \psi_{R}-(d-1) \frac{\partial \Phi}{\partial v_{2}} \frac{\psi}{R} R^{d-1} d R d t=0
$$

which are precisely the weak-form of (3.1).

\subsection{Radial elasticity features}

Upon introducing the velocity, radial strain, and transversal strain, respectively

$$
v=w_{t} \quad a=w_{R} \quad b=\frac{w}{R}
$$

(3.1) is expressed as the first order system

$$
\begin{aligned}
\partial_{t} v & =\frac{1}{R^{d-1}} \partial_{R}\left(R^{d-1} \frac{\partial \Phi}{\partial v_{1}}(a, b, \ldots, b)\right)-\frac{d-1}{R} \frac{\partial \Phi}{\partial v_{2}}(a, b, \ldots, b) \\
\partial_{t} a & =\partial_{R} v \\
\partial_{t} b & =\frac{1}{R} v
\end{aligned}
$$

subject to the constraint $\partial_{R}(R b)=a$, which is again an involution propagating from the initial data and causes no problems as constraint. For $\Phi_{11}:=\frac{\partial^{2} \Phi}{\partial v_{1}^{2}}>0$, the system 3.2 is hyperbolic with wave speeds $\lambda_{ \pm}= \pm \sqrt{\Phi_{11}(a, b, \ldots, b)}, \lambda_{0}=0$.

A comparison with the system of elasticity in one-space dimension (5.1) indicates the following differences 
(i) The system 3.2 has lower order terms and a geometric singularity at the origin.

(ii) As contrasted to the case of one-dimensional elasticity the wave speeds $\lambda_{ \pm}$depend on the lower order terms.

(iii) The system has an involution.

(iv) Following the usual theory of conservation laws a weak entropy solution will be defined to satisfy the entropy inequality

$$
\partial_{t}\left(\frac{1}{2} v^{2}+\Phi(a, b, \ldots, b)\right) R^{d-1}+\partial_{R}\left(R^{d-1} v \frac{\partial \Phi}{\partial v_{1}}(a, b, \ldots, b)\right) \leq 0
$$

expressing the dissipation of mechanical energy. The assumption $\left(\mathrm{H}_{1}\right.$ implies that, in a deviation to the usual theory, the entropy here is merely polyconvex.

Little is known at present concerning the existence of weak solutions for the radial elasticity system (3.2). One additional difficulty is that solutions of (3.2) have to be constructed to satisfy the constraint $\operatorname{det} F>0$ so as to be interpreted as mechanical motions. This constraint is difficult to preserve even in approximating schemes, with the exception of the variational approximation scheme in [9] which preserves the positivity of Jacobians and produces iterates that decrease the mechanical energy.

\section{The problem of cavitation}

The objective is to consider the equations of elasticity

$$
\frac{\partial^{2} y}{\partial t^{2}}=\operatorname{div} \frac{\partial W}{\partial F}(\nabla y)
$$

and to examine under what conditions the equations admit solutions with cavities. As already noted such solutions are radial, $y(x, t)=w(R, t) \frac{x}{R}$ with $R=|x|$, and there will be a cavity provided $w(0, t)>0$, see Figure 2 for a depiction of such a solution.

The natural energy bound for the elasticity system is

$$
\int \frac{1}{2}\left|y_{t}\right|^{2}+|\nabla y|^{p} d x<\infty
$$

with the exponent $p$ determined by the growth of the stored energy $W$. Accordingly, if one is interested to explore cavitating solutions with finite energy, the Sobolev embedding theorem dictates to consider growth exponents $p<d$, where $d$ is the space dimension. Cavitating solutions lie outside the realm of the usual continuum modeling, and one might question if continuum mechanics can account for their presence. Nevertheless, Ball [3] in a groundbreaking work examined whether the equations of equilibrium elasticity admit radial solutions with cavities. He noted that

$$
y(\cdot, t) \in W_{l o c}^{1, p} \Longleftrightarrow\left\{\begin{array}{r}
w(\cdot, t) \text { absolutely continuous on }(0,1) \\
\int_{0}^{1}\left(\left|w_{R}\right|^{p}+\frac{|w|^{p}}{R^{p}}\right) R^{d-1} d R<\infty
\end{array}\right.
$$




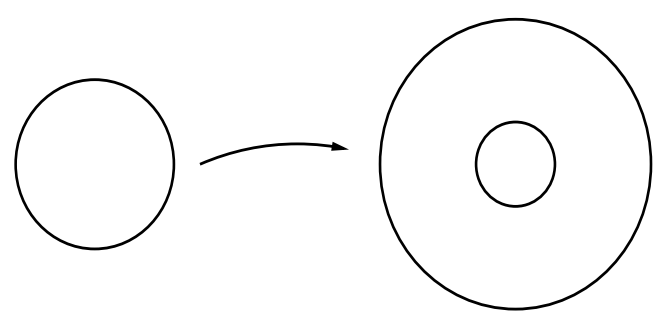

Figure 2: A cavitating solution

and that for $y \in W_{l o c}^{1,1}$ and for $d \geq 2$ there is no delta-mass at the origin associated to the cavity, i.e.

$$
\nabla y=\frac{w}{R} I+\left(w_{R}-\frac{w}{R}\right) \hat{x} \otimes \hat{x} \quad \text { in } \mathcal{D}^{\prime} \text { and a.e. }
$$

This situation should be contrasted with the case $d=1$ where a jump discontinuity induces a delta mass at the origin.

\subsection{Cavitation in equilibrium elasticity}

Ball [3] studied the cavitation problem from two perspectives. Using direct methods of the calculus of variations, he studies the minimization problem

$$
\min \int_{0}^{1} \Phi\left(w^{\prime}(R), \frac{w}{R}, \ldots, \frac{w}{R}\right) R^{d-1} d R
$$

over the set of admissible functions $\mathcal{A}_{\lambda}$. He also considered the associated Euler-Lagrange equations

$$
\begin{aligned}
\partial_{R}\left(R^{d-1} \frac{\partial \Phi}{\partial v_{1}}\right)-(d-1) R^{d-2} \frac{\partial \Phi}{\partial v_{2}} & =0 \\
w(1) & =\lambda
\end{aligned}
$$

and carried out a bifurcation analysis for this system. The analysis in [3] shows:

- There is an absolute minimum in the admissible set

$$
\mathcal{A}_{\lambda}=\left\{w \in W^{1,1}, w(0) \geq 0, w^{\prime}(R)>0, w(1)=\lambda\right\} .
$$

- The minimizer $w_{\lambda}$ satisfies the Euler-Lagrange equations for $R>0$ and

a. There is a critical value $\lambda_{c r}$ such that for $\lambda>\lambda_{c r}$ there is a unique minimizer $w_{\lambda}$ with $w_{\lambda}(0)>0$.

b. The trivial solution $w=\lambda R$ is stable (in a suitable sense) if $\lambda \leq \lambda_{c r}$ and unstable if $\lambda>\lambda_{c r}$. If $\lambda>\lambda_{c r}$ then $w_{\lambda}$ is stable.

The reader is referred to Ball [3] for the details (and precise statements) of these results and to Sivaloganathan and Spector [14] for an account of later results on the problem of cavitation in elastostatics. The problem of cavitation has also been studied in a context of incompressible elasticity [3] which lies outside the realm of our discussion. 


\subsection{Dynamic radial elasticity - Cavitation}

Consider next the problem of dynamic cavitation in compressible, isotropic elastic materials. Such materials have stored energies $W(F)=\Phi\left(v_{1}, v_{2}, v_{3}\right)$ and support an ansatz of radial motions with $w(R, t)$ satisfying the equations of radial elasticity (3.1). The latter admit the special solution $w(R, t)=\lambda R$ which corresponds to a homogeneous elastic deformation of stretching $\lambda$ and is a special solution emanating from initial data $w_{0}(R)=\lambda R$.

Pericak-Spector and Spector in a remarkable work [11, 12] postulated a self-similar ansatz of solutions

$$
y(x, t)=w(R, t) \frac{x}{R}=t \varphi\left(\frac{R}{t}\right) \frac{x}{R}
$$

and constructed a second self-similar solution for the dynamic elasticity system associated to cavity formation $\varphi(0)>0$ provided the stretching $\lambda$ is bigger than some critical value, $\lambda>\lambda_{c r}$.

Theorem 4.1 (Pericak-Spector and Spector [11]) For a stored energy function

$$
\Phi\left(v_{1}, v_{2}, v_{3}\right)=\frac{1}{2}\left(v_{1}^{2}+v_{2}^{2}+v_{3}^{2}\right)+h\left(v_{1} v_{2} v_{3}\right),
$$

with $h: \mathbb{R}_{+} \longrightarrow \mathbb{R}_{+}$satisfying the hypotheses

$$
h^{\prime \prime}>0, \quad h^{\prime \prime \prime}<0 \quad \lim _{v \rightarrow 0} h(v)=\lim _{v \rightarrow \infty} h(v)=\infty,
$$

and for $\lambda>\lambda_{c r}$ there exist cavitating solutions (4.1) for dimension $d=3$ satisfying

$$
\begin{aligned}
w_{t t} & =\frac{1}{R^{2}} \partial_{R}\left(R^{2} \frac{\partial \Phi}{\partial v_{1}}\left(w_{R}, \frac{w}{R}, \frac{w}{R}\right)\right)-\frac{1}{R}\left(\frac{\partial \Phi}{\partial v_{2}}+\frac{\partial \Phi}{\partial v_{3}}\right)\left(w_{R}, \frac{w}{R}, \frac{w}{R}\right), R>0, t>0 \\
w(R, t) & =\lambda R \quad \text { for } R>\bar{r} t \text { for some } \bar{r}>0
\end{aligned}
$$

and $w(0, R)=\lambda R$.

An initial version of this theorem was proved in [11] for $\left(\mathrm{H}_{1}\right]$ and $d \geq 3$ and it was extended in [12] to a far more general class of polyconvex constitutive functions $\Phi$. A variant also holds for dimension $d=2$ but the growth of $\Phi$ is there restricted to be slightly superlinear. Additional regularity properties for the cavitating solutions and a bifurcation study is provided in [10].

We give an outline of the main ideas in the construction and the properties of the constructed solutions. Our exposition follows [11] and [10] and we refer there for the details. The starting point is the self-similar ansatz

$$
w(R, t)=t \varphi\left(\frac{R}{t}\right) \quad s=\frac{R}{t} .
$$

Note that $w(0, t)=t \varphi(0)$ and thus $\varphi(0)$ stands for the velocity of the cavity. Introducing (4.2) to (3.1) leads to the ordinary differential equation

$$
\left(s^{2}-\Phi_{11}\right) \ddot{\varphi}=\frac{2}{s}\left(\dot{\varphi}-\frac{\varphi}{s}\right) \underbrace{\left[\Phi_{12}+\frac{\Phi_{1}-\Phi_{2}}{\dot{\varphi}-\frac{\varphi}{s}}\right]}_{P\left(\dot{\varphi}, \frac{\varphi}{s}, \frac{\varphi}{s}\right) \geq 1} .
$$

An important property is that due to $\left(\mathrm{H}_{1}\right)$ the function $P$ defined above satisfies $P \geq 1$. 


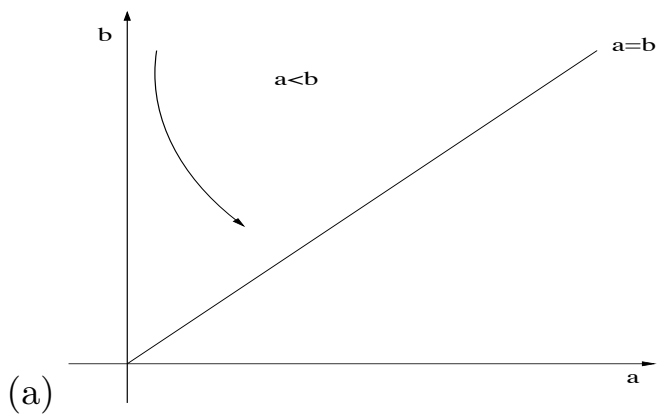

(b)

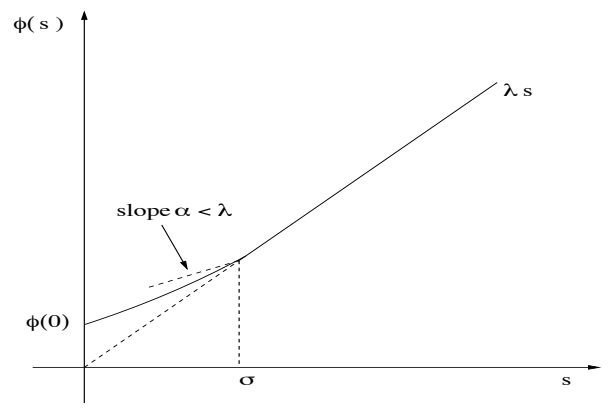

Figure 3: (a) A typical trajectory of 4.4); (b) The cavity solution

It is instructive to rewrite $(4.3)$ as a first order system for the variables

$$
a=\dot{\varphi}, \quad b=\frac{\varphi}{s}
$$

It takes the form

$$
\begin{aligned}
\left(s^{2}-\Phi_{11}(a, b, b)\right) \dot{a} & =\frac{2}{s}(a-b) P(a, b, b) \\
\dot{b} & =\frac{1}{s}(a-b)
\end{aligned}
$$

A simple examination of the system (4.4) reveals the following properties:

- The system is singular with a free boundary type of singularity at the sonic line $s^{2}-\Phi_{11}=0$.

- The system has one class of special solutions, the states $a=b$, corresponding to a homogeneous deformation.

- A second class of solutions consists of jump discontinuities. These satisfy the RankineHugoniot conditions

$$
\begin{aligned}
\sigma^{2}[a]-\left[\Phi_{1}\right] & =0 \\
{[b] } & =0
\end{aligned}
$$

and give rise to shocks. Note that along a shock, $b$ is continuous but $a$ jumps. For functions of class $\left(\mathrm{H}_{1}\right)$, hypothesis $\left(\mathrm{H}_{2}\right)$ implies that $\Phi_{111}<0$ and it can be worked out that the Lax shock admissibility criterion implies that the admissible shocks satisfy $b_{-}=b_{+}$and $a_{-}<a_{+}=\lambda$.

- The main item is to study the third class of solutions to (4.4), that is continuous solutions. The analysis is based on a monotonicity property that can be read directly from (4.4). So long as the solution does not cross the equilibrium diagonal $a=b$ or the sonic singularity, solutions are monotone functions, see Figure 3(a).

The analysis of the continuous solution of $(4.3)$ is based on a de-singularization of the problem. One may introduce the variables $(\varphi, v)$ where $v=\dot{\varphi}\left(\frac{\varphi}{s}\right)^{2}$ and rewrite 4.4 in the form

$$
\begin{aligned}
& \dot{\varphi}=\hat{\Phi}(s, \varphi, v) \\
& \dot{v}=\hat{U}(s, \varphi, v)
\end{aligned} \quad\left\{\begin{array}{l}
\varphi(0)=\varphi_{0}>0 \\
v(0)=v_{0}>0 .
\end{array}\right.
$$


Under $\left(\mathrm{H}_{1}\right)$ the functions $\hat{\Phi}$ and $\hat{U}$ are non-singular at the origin $s=0$ and the standard existence theory can be applied to the last system. At the origin there hold the following properties for the Cauchy stress $T(0)$ (see [11])

$$
\begin{aligned}
\text { either } & T(0)=0 \Leftrightarrow v(0)=H \quad \text { stress free cavity } \\
\text { or } \quad T(0) & =G(\varphi(0)) \quad \text { cavity with content. }
\end{aligned}
$$

The second condition is analogous to the kinetic relations familiar from the theory of phase transitions. We refer to [10] for the corresponding analysis of the singularity at dimension $d=2$.

The constructed solution has the following properties:

(i) $a=\dot{\varphi} \nearrow \quad b=\frac{\varphi}{s} \searrow \quad a-b \nearrow$.

(ii) It can be extended on a maximal interval of existence $(0, T)$

$$
Q=s^{2}-\Phi_{11} \rightarrow 0 \quad a-b \rightarrow c<0 \quad \text { as } s \rightarrow T .
$$

(iii) If $\Phi_{111}<0$ the solution is connected to a uniformly deformed state through a single shock that is admissible via the Lax shock admissibility condition.

We refer to [11] and [10] for the proofs. It should be noted that the constructed cavitating solution consists of a single precursor shock that connects a convex function $\varphi(s)$ to a uniform deformation, see Figure 3(b). It is necessary to have a precursor shock, that is, it is not possible for $\varphi(s)$ to connect in a $C^{1}$-fashion to a uniformly deformed state via a sonic singularity (see [10]). In summary, $\varphi(s)$ looks like:

(a) At $\sigma$ there is a shock.

(b) $\varphi(s)$ convex for $s<\sigma$.

(c) $\varphi(s)=\lambda s$ for $s>\sigma$.

The following remarkable property was proved in [11, Thm 7.2]: The cavitating solution decreases the mechanical energy, namely, if

$$
E\left(y, B_{\rho}\right)=\int_{B_{\rho}} \frac{1}{2}\left|y_{t}\right|^{2}+W(\nabla y) d x
$$

$y_{h}=\lambda x$ is the homogeneous solution and $y_{c}$ is the solution with the cavity, then

$$
\begin{aligned}
& E\left(y_{c}, B_{\rho}\right)-E\left(y_{h}, B_{\rho}\right)=(t \sigma)^{3} \frac{4 \pi}{3}\left[\Phi\left(a_{-}, \lambda, \lambda\right)-\Phi(\lambda, \lambda, \lambda)\right. \\
&\left.+\frac{1}{2}\left(\Phi_{1}\left(a_{-}, \lambda, \lambda\right)+\Phi_{1}(\lambda, \lambda, \lambda)\right)\left(\lambda-a_{-}\right)\right] \\
&<0 \quad \text { whenever } \quad a_{-}:=\varphi(\sigma-)<\lambda
\end{aligned}
$$

where the right hand side in 4.5) corresponds to the dissipation at the precursor outgoing shock and is strictly negative.

This result states nonuniqueness for entropy weak solutions (with polyconvex energy) because of the singularity at the cavity. As already noted in [11, the paradox arises that by opening a cavity the energy of the material decreases, what induces an autocatalytic mechanism for failure. From a perspective of mechanics the problem appears to be that there is no surface energy cost for opening the cavity that is accounted for by the weak solution. 


\section{$5 \quad$ Fracture in 1-d}

Insight into the non-uniqueness issue can be obtained by studying the equations of one-dimensional elasticity or the equivalent form of the elasticity system,

$$
y_{t t}=\partial_{x} \tau\left(y_{x}\right) \longleftrightarrow\left\{\begin{array}{l}
u_{t}-v_{x}=0 \\
v_{t}-\tau(u)_{x}=0
\end{array},\right.
$$

where $v=y_{t}$ is the velocity and $u=y_{x}$ is the strain. This equation admits the special solution $y_{h}(x, t)=\lambda x$ corresponding to a homogeneous deformation with strain $u_{h}=\lambda$ and velocity $v_{h}=0$. We assume that the stress function $\tau(u)$ satisfies the hypotheses

$$
\begin{gathered}
\tau^{\prime}(u)>0, \quad \tau^{\prime \prime}(u)<0 \\
\tau(u) \rightarrow-\infty \quad \text { as } u \rightarrow 0 \quad \text { and } \quad \int_{1}^{u} \tau(s) d s \rightarrow+\infty \quad \text { as } u \rightarrow 0 .
\end{gathered}
$$

Under $\left(a_{1}\right)$ the wave speeds $\lambda_{1,2}(u)= \pm \sqrt{\tau^{\prime}(u)}$ are real and (5.1) is hyperbolic. The hypothesis $\tau^{\prime \prime}(u)<0$ is appropriate for an elastic material exhibiting softening elastic response and plays an important role in the forthcoming analysis. The hypothesis $a_{2}$ is applicable in the case of longitudinal motions and is placed to exclude that a finite volume is compressed down to zero. In the sequel we will consider only tensile deformations and this hypothesis will not play any significant role. (In the case of shearing motions $\tau(u)$ is defined for $u \in \mathbb{R}$ and $\left(a_{2}\right)$ is removed).

Motivated from the problem of cavitation we introduce the ansatz

$$
y(x, t)=t \varphi\left(\frac{|x|}{t}\right) \frac{x}{|x|}=t Y\left(\frac{x}{t}\right)
$$

with $Y(0)>0$. This ansatz is similar to the one used for the solution of the Riemann problem for (5.1) except at the origin $x=0$. A calculation shows that

$$
\partial_{x} y=Y^{\prime}(\xi)+2 Y(0) \delta_{\xi=0}
$$

and such an ansatz could conceivably provide a cavitating (in fact fracturing) solution, except that at the origin there is a delta mass and a suitable interpretation to the term $\tau\left(\partial_{x} y\right)$ should be supplied. Of course this is the main difference between the case $d=1$ and the dimensions $d \geq 2$ where a delta mass in the origin does not appear.

In [8], the following function

$$
y(x, t)=t Y\left(\frac{x}{t}\right) \quad Y(\xi)=\left\{\begin{array}{cl}
\lambda \xi & \xi<-\sigma \\
-Y(0)+\alpha \xi & -\sigma<\xi<0 \\
Y(0)+\alpha \xi & 0<\xi<\sigma \\
\lambda \xi & \sigma<\xi
\end{array}\right.
$$

is tested as a candidate for solution of (5.1). The constants $\alpha, \lambda$ and $Y(0)$ are selected to sastisfy the Rankine-Hugoniot jump conditions at the two outgoing shocks at $\xi= \pm \sigma$

$$
\begin{aligned}
Y(0) & =\sigma(\lambda-\alpha) \\
\sigma^{2} & =\frac{\tau(\lambda)-\tau(\alpha)}{\lambda-\alpha}
\end{aligned}
$$




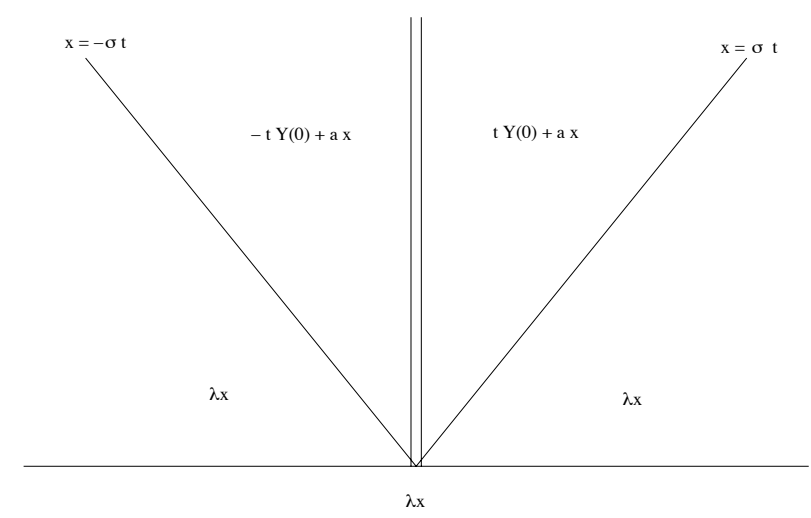

Figure 4: A fracturing motion in Lagrangian coordinates

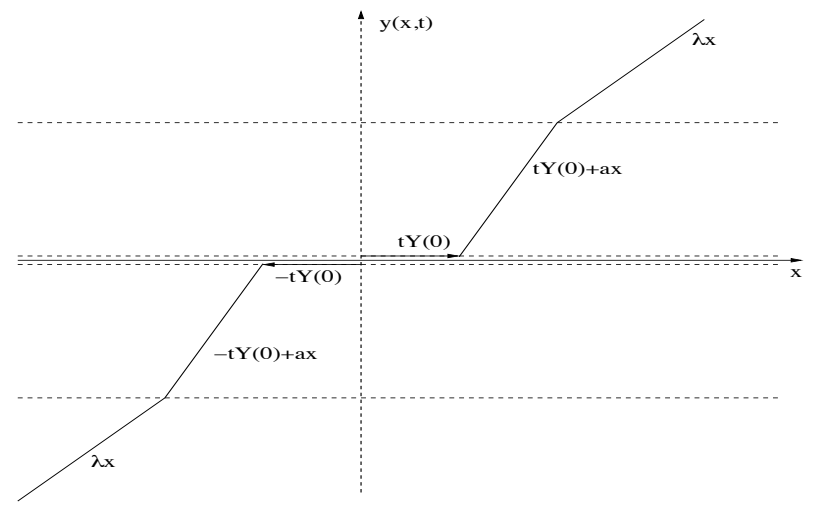

Figure 5: A shear band in Eulerian coordinates

One easily checks that

$$
\tau^{\prime \prime}(u)<0 \text { and } \alpha<\lambda \Longrightarrow \text { both shocks are Lax-admissible. }
$$

The question arises if (5.3) can be given a suitable interpretation as a solution of (5.1). Note that if the equation (5.1) is interpreted as longitudinal motions then such a solution would correspond to fracture, see Figure 4. If (5.1) is interpreted as shear motions then (5.3) corresponds to a shear band, see Figure 5. The difference among the two cases is that the material splits apart in the former case, whereas it remains in contact in the latter. A more precise statement of the underlying question is whether the model (5.1) with the hyperbolic and strain softening stress-strain relation $\left(a_{1}\right)$ may support such motions.

\section{$6 \quad$ Slic-solutions}

There is a class of problems in material science where structures with discontinuous displacement fields emerge via a material instability mechanism. Typical examples are development of cracks in fracture, cavitation in elastic response, or formation of shear bands in plastic deformations. Such problems lie at the limits of applicability of continuum modeling and the usual concept of weak solutions is in any case inadequate to describe these motions. Nevertheless, as the material 
transitions from a regime where continuum modeling is applicable to a regime that the model has to be modified (or perhaps atomistic modeling has to be employed), it is expected that at the interface both types of modeling have to apply in an intermediate regime. It is further expected that such structures should appear in a small parameter limit of more complex models that incorporate "higher-order physics", and that their appearance occurs in a stable way.

The concept of singular limiting induced from continuum solution (in short slic-solution) [8] is an attempt to give meaning to such discontinuous solutions. Roughly speaking a discontinuous solution of (5.1) will be a slic-solution if it can be obtained as the limit of approximate smooth solutions that are an averaging of $y$. Due to the self-similar form of (5.2) averaging in space entails averaging in time. In [8], the following definition is introduced and used to test the example (5.3).

Definition 6.1 Let $y \in L_{l o c}^{\infty}\left((-\infty, \infty) ; L_{\text {loc }}^{1}(\mathbb{R})\right)$ satisfy for some $\varepsilon>0$ the monotonicity condition:

$$
y\left(x_{1}, t\right)-y\left(x_{2}, t\right)>\varepsilon\left(x_{1}-x_{2}\right) \quad \text { for } x_{1}, x_{2}, t \in \mathbb{R} \text { with } x_{1}>x_{2} .
$$

For $\phi$ a mollifier, $\phi \in C_{c}^{\infty}(\mathbb{R}), \phi \geq 0$, supp $\phi \subset B_{1}$ (the ball of radius 1 ), $\int \phi=1$, we let $\phi_{n}=n \phi(n x)$ and define the averaged function

$$
y^{n}(x, t)=\phi_{n} \underset{x}{\star} y=\int \phi_{n}(x-z) y(z, t) d z .
$$

The function $y$ is called a singular limiting induced from continuum (slic-) solution of (5.1) provided for any symmetric mollifier

$$
\partial_{t t} y^{n}-\partial_{x} \tau\left(\partial_{x} y^{n}\right)=: f^{n} \rightarrow 0 \quad \text { in } \mathcal{D}^{\prime}
$$

as $n \rightarrow \infty$.

The example (5.3) is extended to negative values of $t$ by setting $y=\lambda x$ for $t<0$. The resulting function is given in explicit form by

$$
y(x, t)= \begin{cases}\lambda x \mathbb{1}_{x<-\sigma t}+(-t Y(0)+\alpha x) \mathbb{1}_{-\sigma t<x<0}+(t Y(0)+\alpha x) \mathbb{1}_{0<x<\sigma t}+\lambda x \mathbb{1}_{\sigma t<x} & t>0 \\ \lambda x & t<0 .\end{cases}
$$

Using the explicit form, we calculate the averagings in (6.1). A lengthy computation in [8] shows that for $\psi(x, t) \in C_{c}^{\infty}(\mathbb{R} \times \mathbb{R})$

$$
\begin{aligned}
\int_{\mathbb{R}} \int_{\mathbb{R}} y^{n} \psi_{t t}+\tau\left(y_{x}^{n}\right) \psi_{x} d x d t & =\int_{0}^{\infty} \int_{-\frac{1}{n}}^{\frac{1}{n}} \tau\left(\alpha+2 \phi_{n}(x) t Y(0)\right) \psi_{x} d x d t+o(1) \\
& \rightarrow 2 t Y(0) L \int_{0}^{\infty} \psi_{x}(0, t) d t
\end{aligned}
$$

where $L=\lim _{u \rightarrow \infty} \frac{\tau(u)}{u}$. One thus concludes:

If $L>0$ then $y(x, t)$ is not a slic-solution.

If $L=0$ then $y(x, t)$ is a slic-solution. 
Consequently, a meaning to (5.3) as a slic-solution can only be given for sublinear growth of $\tau(u)$.

Next, we consider the energy balance. Let $B=(-r, r)$ contain the entire wave fan of the approximate solution (6.1) at time $t$. The velocity at the boundary of such a domain vanishes, $\left.v^{n}\right|_{\partial B}=0$ and the total energy of the wave fan

$$
E\left[y^{n} ; B\right]=\int_{B} \frac{1}{2}\left(\partial_{t} y^{n}\right)^{2}+W\left(\partial_{x} y^{n}\right) d x
$$

evolves according to the energy balance equation

$$
\frac{d}{d t} \int_{B} \frac{1}{2}\left(\partial_{t} y^{n}\right)^{2}+W\left(\partial_{x} y^{n}\right) d x=\int_{B} f^{n} v^{n} d x
$$

A calculation again shows

$$
\begin{aligned}
\frac{d}{d t} \int_{B} \frac{1}{2}\left(\partial_{t} y^{n}\right)^{2} & +W\left(\partial_{x} y^{n}\right) d x=\int_{B} f^{n} v^{n} d x \\
& =Y(0)^{2} \sigma-2 \sigma(W(\alpha)-W(\lambda))+2 \int_{0}^{\frac{1}{n}} \tau\left(\alpha+2 \phi_{n}(x) t Y(0)\right) 2 Y(0) \phi_{n}(x) d x \\
& \rightarrow \mu_{-\sigma}+\mu_{\sigma}+p_{c}=: T
\end{aligned}
$$

where the total energy production $T$ is split into the energy dissipation of the two shocks $\mu_{ \pm \sigma}$ and the contribution to the energy by the surface energy of the cavity $p_{c}$. One easily also checks that

$$
\begin{aligned}
T & =\mu_{-\sigma}+\mu_{\sigma}+2\left(\tau_{\infty}-\tau(\alpha)\right) Y(0) \\
& =\sigma Y(0)^{2}-2 \sigma(W(\lambda)-W(\alpha))+2 \tau_{\infty} Y(0) \\
& =\sigma Y(0)^{2}+2 Y(0)\left(\tau_{\infty}-\frac{W(\lambda)-W(\alpha)}{\lambda-\alpha}\right)>0
\end{aligned}
$$

so that if $\tau_{\infty}=\infty$ then $T=+\infty$ while if $\tau_{\infty}<\infty$ then $0<T<\infty$.

\section{Cavitation in $3-d$}

Next, we consider solutions of the isotropic elasticity equations (3.1), with stored energy satisfying $\left(\mathrm{H}_{1}\right)$ and $\left(\mathrm{H}_{2}\right)$, of the form

$$
y(x, t)=w(|x|, t) \frac{x}{|x|}=t \varphi(s) \frac{x}{|x|}, \quad s=\frac{|x|}{t}
$$

where $\varphi(s)$ is the cavitating self-similar solution outlined in section 4 . The objective is to examine how the intuition from the example in section 5 transfers to the problem of cavitation.

The following extension of the notion of slic-solution - adapted to the radial case - can be introduced.

Definition 7.1 Let $y(x, t)=w(|x|, t) \frac{x}{|x|}$ with $w \in L_{l o c}^{\infty}\left(\mathbb{R} ; L_{l o c}^{1}(\mathbb{R})\right)$ and $w(\cdot, t)$ monotone increasing satisfy $y(x, t)=\lambda x$ for $t \leq 0$ and for $|x|>\bar{r} t, t>0$ or some $\bar{r}>0$. The function $y$ is called $a$ singular limiting induced from continuum (slic)-solution of 2.1) if

$$
y^{n}=w^{n}(|x|, t) \frac{x}{|x|}, \quad \text { with } \quad w^{n}=\phi_{n} \underset{R}{*} w,
$$


satisfies $\operatorname{det} \nabla y^{n} \geq \varepsilon_{n}>0$ for all $\in \mathbb{N}$, and

$$
\frac{\partial^{2} y^{n}}{\partial t^{2}}-\operatorname{div} \frac{\partial W}{\partial F}\left(\nabla y^{n}\right)=: f^{n} \rightarrow 0 \quad \text { in } \mathcal{D}^{\prime}
$$

as $n \rightarrow \infty$, for all $\phi \in C_{c}^{\infty}(\mathbb{R})$ positive, symmetric mollifiers with $\phi(0)>0$.

The convolution $w^{n}=\phi_{n} \underset{R}{\star w}$ is defined by first extending $w(R, t)$ antisymmetrically in $R$ and the definition takes into account the layer structure at the cavity. In agreement with the definition of slic-solution (and of the energy in one space dimension) we define the energy of a multidimensional slic-solution.

Definition 7.2 Let $w \in W_{\text {loc }}^{1, \infty}\left(\mathbb{R} ; L_{\text {loc }}^{1}(\mathbb{R})\right)$, The energy of a slic-solution in a domain $B \subset \mathbb{R}^{d}$ containing the entire wave fan and for a.e. $t \in \mathbb{R}$ is defined as

$$
E[y, B](t):=\lim _{n \rightarrow \infty} \int_{B} \frac{1}{2}\left|y_{t}^{n}(x, t)\right|^{2}+W\left(\nabla y^{n}(x, t)\right) d x
$$

with $y^{n}$ defined by 7.1 .

The following is proved in [8, Thm 3.8, Prop 3.10] based on detailed estimations of $w^{n}=\phi_{n} \star R_{R} w$ on the entire wave fan but mainly around the cavity:

Theorem 7.3 (i) The weak solution constructed by Pericak-Spector and Spector is a slic-solution if and only if

$$
\lim _{u \rightarrow \infty} \frac{h^{\prime}\left(u^{3}\right)}{u}=0
$$

(ii) If $L=\lim _{u \rightarrow \infty} \frac{h(u)}{u}$ then

$$
\begin{aligned}
\lim _{n \rightarrow \infty} \int_{B} \frac{1}{2}\left|v^{n}\right|^{2}+W\left(\nabla y^{n}\right)-W(\lambda) d x & \\
& =\underbrace{\int_{B}\left[\frac{1}{2}|v|^{2}+W(\nabla y)-W(\lambda)\right] d x}_{<0}+\underbrace{(t \varphi(0))^{3} \frac{4 \pi}{3} L}_{>0}=: P_{w f}>0 .
\end{aligned}
$$

In particular, (ii) shows that for a slic-solution the energy of the solution with cavity is larger than the energy of the uniform deformation.

A rough explanation of the discrepancy. We refer to [8] for the proof, but outline the main calculation of the discrepancy between a weak and a slic solution. Recall that

$$
\begin{aligned}
y(x, t)=w(R, t) \frac{x}{R} & =t \varphi\left(\frac{R}{t}\right) \frac{x}{R} \\
v(R, t) & =w_{R}\left(\frac{w}{R}\right)^{2}
\end{aligned}
$$

where $\varphi(s)$ is the self-similar solution of (4.3) in section 4 , and that the specific volume $v(\cdot, t)$ is strictly monotone increasing and obeys the bounds

$$
v_{0} \leq v(R, t) \leq \lambda^{3} .
$$


The approximate solution and the corresponding approximation of the specific volume are

$$
\begin{aligned}
y^{n}(x, t) & =w^{n}(R, t) \frac{x}{R} \\
w^{n}(R, t) & =\phi_{n} \star w(\cdot, t) \\
v^{n} & =w_{R}^{n}\left(\frac{w^{n}}{R}\right)^{2}
\end{aligned}
$$

where $\phi_{n}$ symmetric mollifier, $\phi(0)>0$ and $w(\cdot, t)$ is an odd extension of $w$. It can be proved that $v^{n}$ satisfies the bounds

$$
\begin{aligned}
& c_{1} \leq v^{n}(R, t) \leq c_{2} \quad \text { for } R>\frac{1}{n} \\
& c_{\phi} n^{3} w(0, t)^{3} \leq v^{n}(R, t) \leq c_{3}\left(1+t^{3} n^{3}\right) \quad \text { for } R<\frac{1}{n}
\end{aligned}
$$

where $c_{\phi}$ a positive constant depending on the shape of the mollifier. Note that the approximate specific volume $v^{n}$ detects that there is a cavity forming, while $v$ stays bounded away from zero.

To show we have a slic-solution we need to show that

$$
\frac{\partial^{2} y^{n}}{\partial t^{2}}-\operatorname{div} \frac{\partial W}{\partial F}\left(\nabla y^{n}\right)=: f^{n} \rightarrow 0 \quad \text { in } \mathcal{D}^{\prime}
$$

The discrepancy $D$ between the weak and the slic-solution lies in the behavior near the cavity, in the ball $|x|<\frac{1}{n}$, and can be computed by the following heuristic "calculation"

$$
\begin{aligned}
D & =\iint S\left(\nabla y^{n}\right): \nabla \psi \mathbb{1}_{|x|<\frac{1}{n}} d x d t \\
& \sim \iint_{|x|<\frac{1}{n}}\left(\frac{w^{n}}{R}\right)^{2} h^{\prime}\left(v^{n}\right)|\nabla \psi| R^{2} d R d t \\
& \sim \iint_{0}^{1} \frac{h^{\prime}\left(n^{3}\right)}{n}|\nabla \psi| \rho^{2} d \rho d t .
\end{aligned}
$$

Therefore, $D$ is related to the $\operatorname{limit}_{u \rightarrow \infty} \frac{h^{\prime}\left(u^{3}\right)}{u}$. For the details and the calculation of the discrepancy in the energy, see [8].

In conclusion, two issues may arise when a weak solution is viewed as limit of continuous solutions in a context of strong singularities (like a cavity or fracture or shear band)

a. New terms might appear in the momentum balance equation.

b. Even when such forces vanish in the limit, their effect can be felt in the energy balance and their contribution might affect the total energy as the singularity forms.

Acknowledgements This research was supported by the EU FP7-REGPOT project "Archimedes Center for Modeling, Analysis and Computation". AET is partially supported by the "Aristeia" program of the Greek Secretariat for Research. 


\section{References}

[1] J.M. BALL, Convexity conditions and existence theorems in nonlinear elasticity, Arch. Rational Mech. Anal. 63 (1977), 337-403.

[2] J.M. Ball, J.C. Currie And P.J. Olver Null Lagrangians, weak continuity, and variational problems of arbitrary order J. Functional Analysis 41 (1981), 135-174.

[3] J.M. BALl, Discontinuous equilibrium solutions and cavitation in nonlinear elasticity, Philos. Trans. Roy. Soc. London Ser. A, 306, (1982) 557-611.

[4] C. Dafermos, Quasilinear hyperbolic systems with involutions, Arch. Rational Mech. Anal. 94 (1986), 373-389.

[5] S. Demoulini, D.M.A. Stuart, A.E. Tzavaras, A variational approximation scheme for three-dimensional elastodynamics with polyconvex energy, Arch. Rational Mech. Anal. 157 (2001), 325-344.

[6] D.G.B. Edelen, The null set of the Euler-Lagrange operator Arch. Rational Mech. Anal. 11 (1962), 117-121.

[7] J.L. Ericksen, Nilpotent energies in liquid crystal theories, Arch. Rational Mech. Anal. 10 (1962), 189-196.

[8] J. Giesselmann and A.E. Tzavaras, Singular limiting induced from continuum solutions and the problem of dynamic cavitation. (submitted), (2013), arXiv preprint arXiv:1306.6084.

[9] A. Miroshnikov and A.E. Tzavaras, A variational approximation scheme for polyconvex elastodynamics that preserves the positivity of Jacobians. Comm. Math. Sciences 10 (2012), $87-115$.

[10] A. Miroshnikov and A.E. Tzavaras, On the construction and properties of weak solutions describing dynamic cavitation. (preprint).

[11] K.A. Pericak-Spector and S.J. Spector, Nonuniqueness for a hyperbolic system: cavitation in nonlinear elastodynamics. Arch. Rational Mech. Anal. 101 (1988), 293 - 317.

[12] K.A. Pericak-Spector and S.J. Spector, Dynamic cavitation with shocks in nonlinear elasticity. Proc. Royal Soc. Edinburgh Sect A 127 (1997), 837 - 857.

[13] T. Qin, Symmetrizing nonlinear elastodynamic system, J. Elasticity 50 (1998), 245-252.

[14] J. Sivaloganathan and S.J. Spector, Myriad radial cavitating equilibria in nonlinear elasticity. SIAM J. Appl. Math. 63 (2003), 1461 - 1473.

[15] C. Truesdell, W. Noll, The non-linear field theories of mechanics, Handbuch der Physik III, 3 (Ed. S.Flügge), Springer Verlag, Berlin, 1965.

[16] D.H. Wagner, Symmetric hyperbolic equations of motion for a hyper-elastic material, $J$. Hyper. Differential Equations 6 (2009), 615-630. 